\title{
Integração de preços nas principais praças de comercialização do milho em grão no Brasil
}

\author{
Maykon Daniel Gonçalves Silva \\ Universidade Federal de Goiás. \\ E-mail: maykondaniel@hotmail.com
}

\section{Cleyzer Adrian da Cunha}

Professor da Universidade Federal de Goiás.

E-mail: cleyzercunha@gmail.com

\author{
Alcido Elenor Wander \\ Embrapa Arroz e Feijão (CNPAF) \\ E-mail: alcido.wander@embrapa.br
}

Resumo: O objetivo deste estudo foi analisar a integração de preços no mercado brasileiro de milho em grão, considerando os custos de transação. Foram analisados preços diários do milho em grão no período de janeiro de 2009 a dezembro de 2016, nos mercados físicos de Sorriso (MT), Dourados (MS), Paranaguá e Cascavel (PR), Rio Verde (GO), Passo Fundo (RS), Triângulo Mineiro (MG) e Campinas (SP). São regiões de maior dinâmica na comercialização do grão no País. A metodologia consistiu no teste de raiz unitária, cointegração de Johansen, estimação do modelo vetorial de correção de erro com threshold (TVEC) dois regimes. Os resultados indicam que Campinas possui uma relação de equilíbrio de longo prazo com os demais mercados e a estimação do modelo TVEC confirma a presença de um threshold. Nos regimes Down, onde a maior parte da porcentagem da amostra se encontra, as variações de preços ocorridas em Campinas afetaram os preços de Dourados e Triangulo Mineiro somente no curto prazo, ou seja, variações de preços passados. Nos regimes Up as variações de preços em Campinas afetaram em curto e em longo prazo os demais mercados físicos. Além disso, os parâmetros threshold, indicam a existência de custos de transação nas inter-relações entre os preços. De modo que um choque de preços resultado por excesso de demanda em Campinas, por exemplo, superior a 1,22\%; 1,45\%; 1,26\%; 0,9\%; 1,27\%; 1,03\% e 1,39\% promovem um ajustamento de preços em Cascavel, Dourados, Paranaguá, Passo Fundo, Rio Verde, Sorriso e Triângulo Mineiro, respectivamente.

Palavras-chave: integração, preços, milho, Brasil

Abstract: The paper examines the integration of prices in the Brazilian corn-grain market, considering the transaction costs. Daily grain prices were analyzed from January 2009 to December 2016, in the physical markets of Sorriso (MT), Dourados (MS), Paranaguá and Cascavel (PR), Rio Verde (GO), Passo Fundo (RS), Triângulo Mineiro (MG) and Campinas (SP). The methodology consisted of the unit root test, Johansen cointegration, estimation of the vector error correction model with threshold (TVEC), two regimes. The results indicate that Campinas has a long-term equilibrium relation with the other markets and the estimation of the TVEC model confirms the presence of a threshold. In addition, the threshold parameters indicate the existence of transaction costs in the interrelations between prices. So that a price shock resulting from excess demand in Campinas, for example, exceeds 1.22\%; 1.45\%; 1.26\%; 0.9\%; 1.27\%; 1.03\% and 1.39\% promote a price adjustment in Cascavel, Dourados, Paranaguá, Passo Fundo, Rio Verde, Sorriso and Triângulo Mineiro, respectively.

Key words: integration, prices, corn, and Brazil

JEL Code: Q13 


\section{INTRODUÇÃO}

O milho é a segunda cultura mais importante na agricultura brasileira em termo de volume de produção, atrás apenas da soja. A produção desse grão esteve em crescente ascensão nas últimas décadas, concentrando-se principalmente nas regiões Sul, Sudeste e Centro-Oeste. Vem sendo produzido principalmente nos estados de Mato Grosso, Paraná, Mato Grosso do Sul, Goiás, Minas Gerais, Rio Grande do Sul e São Paulo. Conforme o quinto levantamento no mês de fevereiro feito pela Conab (2017a), esses estados representam 87,11\% da produção nacional na safra 2015/16.

A produção no Brasil tem uma perspectiva de crescimento para os próximos anos. De acordo com BRASIL (2016) em estudo sobre projeções do agronegócio no Brasil a produção é projetada em 94,7 milhões de toneladas de milho até 2025/26. Tal aumento é graças às condições climáticas favoráveis, tecnologia aplicada, características edafoclimáticas, que permitem a utilização para plantio de mais de uma cultura por ano, na mesma área. Possibilitado o binômio soja/milho com uso máximo de máquinas e implementos agrícolas (CONAB, 2015).

Internacionalmente, a demanda de milho deve aumentar. Os principais consumidores são os EUA, China, União Europeia, Brasil, México e a Rússia que teve sua demanda dobrada devido a um forte uso de ração animal à base de milho. Os preços internacionais têm uma projeção de aumento na bolsa de Chicago, o que deve estimular mais ainda a produção (CONAB, 2015).

Nacionalmente, o principal destino da produção do milho é atender as demandas dos setores avícolas e suinícolas. Tais setores são responsáveis por 45,52\% do consumo total do grão. Somando ao setor da bovinocultura, a demanda por milho aumenta para 50,10\% na safra 2015/16 (ABIMILHO, 2016).

Por se constituir um dos principais produtos no país e dadas às mudanças ocorridas na cadeia produtiva no que se refere à oferta e a demanda, no qual impacta de modo significativo no comportamento dos preços, estudos de integração espacial do milho são bem relevantes.

O estudo de integração em diferentes mercados é amplamente aplicado ao contexto agrícola, a qual analisa a informação contida nos preços de determinada commodities que é transmitida de uma região a outra (ALVES; LIMA, 2010).

Confirmado por Sousa, Braga e Cunha (2010), o estudo sobre integração é importante na qual busquem as interdependências dos preços de diferentes mercados de dada mercadoria. Vários métodos econométricos são usados para analisar a integração de mercados separados. Conforme Mattos, Lima e Lirio (2009), a cointegração é uma técnica amplamente utilizada por fornecer um tratamento adequado às séries não-estacionárias e passou a ser a principal e, muitas vezes, a única ferramenta empregada nas análises de integração de mercados.

É importante observar que a existência de custos de transação prejudica a comercialização, logo, as possibilidades de arbitragem, o que dificulta a integração entre mercados. Segundo Mattos et al. (2010) tais custos geram faixas nas quais os ajustamentos de preços ocorrem com diferentes intensidades, a depender da magnitude 
p. 57 - Integração de preços nas principais praças de comercialização do milho em grão no Brasil

desses custos, essas faixas são denominadas regimes, que são delimitados por limiares ou thresholds.

Dessa maneira, esse estudo busca responder a seguinte pergunta de pesquisa: Qual o grau de integração espacial do mercado do milho em grão entre as principais regiões produtoras, considerando a existência de custo de transação?

O objetivo geral desse estudo é analisar a integração de preços no mercado brasileiro de milho, considerando a presença de custos de transação, no período de janeiro de 2009 a dezembro de 2016. Especificamente é examinar a existência de equilíbrio de longo prazo entre os pares de preços das principais regiões produtoras de milho e determinar o parâmetro threshold que representa o custo de transação entre as principais regiões produtoras de milho.

A estrutura do artigo está dividida em cinco partes. O primeiro é esta introdução com uma breve explanação sobre as perspectivas do mercado do milho em grão e o tema de integração de mercado, juntamente com o problema de pesquisa e objetivos propostos. Em seguida apresenta-se à revisão de literatura, que explica os conceitos de integração de mercado e custo de transação. Na terceira parte trata dos procedimentos metodológicos de Dickey-Fuller Aumentado (ADF), teste de cointegração de Johansen e modelo TVEC com dois regimes. Na quarta são apresentados os resultados obtidos. Por fim, são apresentadas as considerações finais.

\section{INTEGRAÇÃO DE MERCADO}

O estudo de mercados separados espacialmente que se relacionam por meio do fluxo de bens, informação e preço é conhecido na literatura como integração de mercado. Segundo Meyer (2004), a integração de mercado é definida como o grau de transmissão de preço entre mercados relacionados verticalmente ou espacialmente.

Dessa maneira quanto maior o grau de integração, maior será a transmissão de preços (ALVES, 2009). Uma definição semelhante se encontra em Goodwin e Piggott (2001), segundo o qual a integração de mercado pode ser descrita como choques que são transmitidos entre os mercados espacialmente separados. Os autores explicam que a integração de mercado possibilita o descobrimento de preços e o funcionamento do mercado uma vez que desvios persistentes de integração podem implicar em risco nas possibilidades de lucros dos arbitradores.

Os choques podem ser entendidos como mudanças na estrutura da demanda e oferta de um mercado que afeta os preços. Conforme Fackler e Goodwin (2001) a integração de mercado pode ser medida por uma razão de preços entre duas regiões, onde um choque de demanda ou oferta de uma região é transmitido à outra região.

$$
R_{i, j}=\frac{\partial P_{j} / \partial \varepsilon_{i}}{\partial P_{i} / \partial \varepsilon_{i}}=\frac{\partial P_{j}}{\partial P_{i}}
$$


O choque na região $i$ é representado por $\varepsilon_{i}$, a variação infinitesimal do choque econômico que afeta o preço do bem na região $j$ é $\partial P_{j} / \partial \varepsilon_{i}$, já a variação infinitesimal do choque que altera o preço na região $i$ é $\partial P_{i} / \partial \varepsilon_{i}$. De modo que $R_{i, j}$ é a razão de transmissão de preço da região $i$ para a região $j$.

A perfeita integração do mercado pode ocorrer se a relação de transmissão de preços esperado é igual a 1 . Note que a relação pode não ser simétrica (i.e., $R_{i, j} \neq R_{j, i}$ ), de modo que é possível para uma região ser mais integrada com outra região do que é o outro com ele (FACKLER; GOODWIN, 2001).

Uma definição de integração de mercado mais ampla é dada por González-Rivera e Helfand (2001) que abrangem duas dimensões sobre integração, a saber: comércio e informação. Ocorre integração de mercado se o conjunto de localidades compartilha ou negocia o mesmo produto e as mesmas informações de longo prazo. A segunda condição em uma abordagem de cointegração equivale a uma existência de um fator de integração comum a todas as séries de preços, ou seja, uma movimentação conjunta dos preços.

A ocorrência ou existência de integração entre mercados é garantida principalmente pelos mecanismos de troca espacial e arbitragem (GOODWIN; PIGGOTT, 2001). O conceito de arbitragem é o ato de comprar uma mercadoria em um local, tempo ou forma em que ela é mais barata e vendê-la onde ela for mais cara, após compensados os custos de transação (PEREIRA, 2005). De maneira que as ações dos arbitradores espaciais assegurarão que os preços de um bem homogêneo, em quaisquer duas localidades, diferirão, no máximo, ao custo de transportar a mercadoria de uma região com menor preço para outra de maior preço (ROSADO, 2006).

De acordo com Fackler e Goodwin (2001), arbitragem espacial pode ser expressa na relação:

$$
P_{i}-P_{j} \leq T_{j, i}
$$

Onde $P_{i}$ representa o preço da commodity na região i, $P_{j}$ representa o preço da commodity na região j e $T_{j, i}$ representa os custos para mover a commodity da região j para a região i.

Segundo Pereira (2005) a expressão (2) é denominada condição de arbitragem espacial e constitui-se um conceito de equilíbrio. Assim, conforme Campenhout (2007) os mercados são ditos integrados se eles estão ligados por um processo de arbitragem.

Deve-se ressaltar que não há na literatura, uma terminologia uniforme para o termo $T_{j, i}$ que aparece na expressão (2). Normalmente, são três os termos utilizados: custos de transportes, custos de transferências e custos de transação (MATTOS, 2008). Neste trabalho será comum se referir a custos de transação.

\section{CUSTO DE TRANSAÇÃO E INTEGRAÇÃO DE MERCADO}

Custos de transação são os custos de negociar, redigir e garantir o cumprimento de um contrato, e a unidade básica de análise quando se trata de custos de transação seria o contrato (FIANI, 2002). 
A ideia de custos de transação surgiu com artigo "The nature of the firm" de Ronald Coase (1937). Segundo ele, custos de transação é o custo de utilizar o mecanismo de preços; são os custos de organizar a produção através do sistema de preço; é o custo de negociação e celebração de um contrato de separação para cada transação, assim a empresa surge com o intuito de reduzir esse custo, apesar de não o eliminar.

O trabalho de Ronald Coase (1937) inspirou os avanços que dão sustentação à "Nova Economia Institucional", que é uma perspectiva econômica que busca ampliar a economia focando normas sociais e legais, e regras que embasam a atividade econômica. A economia dos custos de transação faz parte desse arcabouço teórico (MACHADO; WANDER; SILVA, 2010).

As principais dimensões em que a economia de custo de transação se baseia para descrever as transações são: a frequência com que elas acontecem, o grau e tipo de incerteza a que estão sujeitas e a condição de especificidade de ativos (WILLIAMSON, 1996).

Especificidade dos ativos refere-se a um investimento especializado que não pode ser reafetado a usos alternativos ou por usuários alternativos, exceto em perda de valor produtivo. A especificidade de ativos pode assumir várias formas, das quais os recursos humanos, físicos, locais e dedicados são os mais comuns. Ativos específicos dão origem à dependência bilateral, o que complica as relações contratuais. Consequentemente, tais investimentos nunca seriam feitos a não ser para contribuir para reduções prospectivas nos custos de produção ou adições à receita (WILLIAMSON, 1996).

Frequência possui a característica que está associada ao número de vezes que dois agentes realizam determinadas transações, que podem ocorrer uma única vez, ou se repetir dentro de uma periodicidade conhecida (ZYLBERSZTAJN; NEVES, 2000).

Incerteza é quando a impossibilidade de previsão de choques que possam alterar as características dos resultados da transação não permite que os agentes que dela participam desenhem cláusulas contratuais que associem a distribuição dos resultados aos impactos externos, uma vez que estes não são conhecidos ex ante (ZYLBERSZTAJN; NEVES, 2000).

Além disso, conforme Williamson (1996) os agentes apresentam comportamentos oportunistas e racionalidade limitada. Racionalidade limitada se refere a um comportamento que é intencionalmente racional, mas apenas de forma limitada. É uma condição de competência cognitiva limitada para receber, armazenar, recuperar e processar informações. Todos os contratos complexos são inevitavelmente incompletos por causa dos limites da racionalidade.

Oportunismo é o auto interesse buscando com astúcia, para incluir esforços calculados para enganar, ofuscar, e de outra forma confundir. O oportunismo deve ser distinguido da simples busca de interesse próprio, de acordo com a qual os indivíduos jogam um jogo com regras fixas que obedeçam de forma confiável.

Diante disso, a definição de custos de transação segundo Williamson (1996) são os custos de funcionamento do sistema econômico. Os custos podem ser ex ante e ex post; os custos ex ante são os de elaboração, negociação e salvaguarda de um acordo; e 
os custos ex post são os de ajustamentos e adaptação que surgem quando a execução do contrato é desalinhada devido a lacunas, erros, omissões e perturbações imprevistas.

Custos de transação aumentam às possibilidades de os mercados serem segmentados, tornando maiores às chances da região possuir suas próprias ofertas e demandas locais, por isso, mesmo sendo duas regiões integradas, o fato de ser caro transferir bens e informações implica que ajustamentos aos choques de oferta e demanda levará mais tempo, dessa maneira, quanto menor os custos de transação, maior a integração dos mercados (MATTOS, 2008).

Do mesmo modo, sabe-se que arbitragem ocorre quando os diferenciais de preços entre dois mercados separados espacialmente são maiores que os custos de transação. Dessa forma, quanto maiores for os custos de transação menor a possibilidade de arbitragem e consequentemente menor o grau de integração entre mercados.

Exemplificando as condições de arbitragem espacial na presença de custos de transação, consideram-se dois mercados separados espacialmente, $A$ e $B$, que comercializam um bem homogêneo. O custo de transação no tempo $t$ entre os mercados A e B é $K_{t}^{A B}$, e os preços correntes podem ser expressos por $P_{t}^{A}$ e $P_{t}^{B}$. Considera-se também ganhos com arbitragem. Ocorre transação do mercado $A$ para o $B$, se a relação $P_{t}^{A}+K_{t}^{A B} \leq P_{t}^{B}$ é mantida. Não há arbitragem quando $P_{t}^{A}+K_{t}^{A B}>P_{t}^{B}$ no entanto, somente quando $P_{t}^{A}+K_{t}^{A B} \geq P_{t}^{B}$ pode-se dizer que estes dois mercados estão integrados desde que $P_{t}^{A}+K_{t}^{A B}<P_{t}^{B}$ no longo prazo, dado ausência de comércio ou se há falha de mercado devido um abundância de mercadorias (BALCOMBE; BAILEY; BROOKS, 2007).

De acordo com Rosado (2006), custos de transação podem ser excessivamente altos devido a uma variedade de razões, como resultado da ineficiência na execução dos contratos, de proteção policial insuficiente, corrupção, impostos excessivamente altos e infraestrutura de transporte e de comunicações inadequada.

Para verificar a integração de mercados separados espacialmente são empregados vários métodos, desde modelos bem simples que calculam a correlação entre preço a modelos bem mais sofisticados que incluem custos de transação (ALVES; LIMA, 2010).

Muitos estudos sobre integração de mercados não inclui os custos de transação em suas análises, e uma das principais dificuldades é a obtenção de dados de custos de transação. Com isso surgiram diversas metodologias novas com o intuito de incluir os custos de transação através de uma estimação de um threshold.

Alguns estudos apontam a necessidade de inclusão de threshold para representar o custo de transação são encontrados em Goodwin e Piggott (2001); Sephton (2003); Meyer (2004); Cunha, Scalco e Wander (2013).

\section{FONTE DOS DADOS}

O estudo corresponde às regiões formadoras de preço do milho nos estados do Mato Grosso, Mato Grosso do Sul, Goiás, Paraná, Minas Gerais, São Paulo e Rio Grande 
p. 61 - Integração de preços nas principais praças de comercialização do milho em grão no Brasil

do Sul, representado por Sorriso, Dourados, Rio Verde, Cascavel e Paranaguá, Triângulo Mineiro, Campinas e Passo Fundo, respectivamente. São as praças que apresentam as maiores produções e para as quais existem dados disponíveis. Os dados coletados correspondem ao período de 02 de janeiro de 2009 a 29 de dezembro de 2016, totalizando 1997 observações.

As séries de preços nominais e diárias do milho estão em $\mathrm{R} \$$ por saca de $60 \mathrm{~kg}$, à vista, em nível de atacado sem Imposto sobre Circulação de Mercadorias e Serviços (ICMS). Os dados foram obtidos no Centro de Estudos Avançados em Economia Aplicada (CEPEA).

Nas estimativas empíricas, foram utilizadas as rotinas do software $R$ por meio dos logaritmos das séries, e o modelo estimado foi threshold error correction models (TVEC).

\section{MODELO VETORIAL DE CORREÇÃO DE ERROS COM THRESHOLDS}

O modelo vetorial de correção de erro é uma versão mais completa do modelo Vetorial Autorregressivo (VAR), à medida que incluem um termo de correção do erro que expressa os desvios de curto prazo na relação de equilíbrio de longo prazo entre as variáveis do modelo, ou seja, quando as variáveis são cointegradas elas podem ser representadas na forma de um modelo vetorial de correção de erros (VEC).

O uso de modelos VEC é muito utilizado em estudos de integração de mercado onde não se sabe qual preço causa o outro, de maneira que os ajustamentos de preços resultado dos desvios no equilíbrio de longo prazo são assumidos ser função linear e contínua e, portanto, desvios muito pequenos no equilíbrio de longo prazo sempre levarão a um processo de ajustamento de preço em cada mercado (MEYER, 2004).

No entanto, a realidade inclui custos de transação entre mercados. Dessa forma, hipóteses que não considerarem custos de transação, leva a resultados tendenciosos. Logo, o ajustamento ao equilíbrio de longo prazo não deverá ser contínuo em casos de custos de transação significativos (MEYER, 2004).

A incorporação dos efeitos dos custos de transação fornece uma justificativa para a utilização de modelos de ajustamento de preços como inclusão da variável representativa do efeito threshold (MATTOS, 2008).

De acordo com Goodwin e Piggott (2001), a incorporação desse efeito threshold aos modelos VEC, dão origem ao que é conhecido, na literatura especializada, como modelos TVEC (modelos vetoriais de correção de erro com threshold).

Diante disso, muitos estudos passaram a considerar os efeitos de um custo de transação, representados por um parâmetro threshold. Neste caso, cita-se Balke e Fomby (1997) que realizaram os primeiros estudos que combinam não linearidade e cointegração.

Conforme Sephton (2003), a cointegração inicialmente assume uma relação linear e estável ao longo do tempo, mas a presença de um threshold permite a relação de equilíbrio mudar se a série exibe comportamento diferente de um threshold. 
As vantagens de utilizar um modelo TVEC residem no fato de não se saber previamente a relação de causalidade entre preços nos diferentes mercados. Além disso, possibilita que seja identificada a trajetória temporal do processo de transmissão de preço e, portanto, a integração de mercado (JUSTEN JUNIOR, 2013).

De acordo com Tardelli (2012), em um modelo TVEC o número de regimes ( $\mathrm{j}=1,2)$ será definido de acordo com a significância da não linearidade, sendo que a variável threshold é simbolizada por $E C T_{t-1}$ e o parâmetro de threshold por $\gamma$. Formalmente o modelo TVEC pode ser expresso da seguinte forma:

$\Delta P_{t}=\left\{\begin{array}{l}\delta^{(1)}+\sum_{i=1}^{k-1} \Gamma_{i}^{(1)} \Delta P_{t-i}+\alpha^{(1)} E C T_{t-1}+v_{t}^{(1)}, \text { se }\left|E C T_{t-1}\right| \leq \gamma \\ \delta^{(2)}+\sum_{i=1}^{k-1} \Gamma_{i}^{(2)} \Delta P_{t-i}+\alpha^{(2)} E C T_{t-1}+v_{t}^{(2)}, \text { se }\left|E C T_{t-1}\right|>\gamma\end{array}\right\}$

Segue:

Pt é o logaritmo natural dos preços dos mercados em diferentes regiões;

$\delta(j)$ vetor de coluna $(2 \times 1)$;

$\lceil i(j)$ matriz de parâmetro (2x2);

$\alpha(j)$ vetor de coluna (2x1) dos coeficientes de ajustamento;

$E C T_{t-1}$ termo de correção de erros

$v t(j)$ termo de erro.

O regime 1 é o espaço onde os reajustes de preços em valores absolutos estão abaixo do ponto limiar, na qual não ocorre transmissão de preço. No regime 2 é o espaço em que os reajustes de preços ocorrem, representando a zona de transmissão de preço.

Conforme Cunha, Silva Neto e Scalco (2014), a banda entre dois threshold, o qual se tem regime acima e abaixo do termo de equilíbrio de longo prazo, pode ser interpretado como desvios em torno do equilíbrio de longo prazo, que são comparados com custo de ajustamento. Ou seja, os desvios do equilíbrio de longo prazo são tão pequenos que não vão levar a um ajuste de preços relativos.

Apesar da presença da região de não ajustamento, não significa que os mercados não sejam integrados. Pois de acordo com Mattos (2008), isso pode apenas representar a região que não ocorre uma integração entre os preços, o que é justamente resultado da presença dos custos de transação e das condições de arbitragem.

Por fim, três passos são propostos por Meyer (2004) para estimação de um TVEC. Primeiramente, são testadas a estacionariedade das series de preços, por meio do teste de raiz unitária Dickey e Fuller (1979) aumentado. A seguir, verificado a estacionariedade das series, realiza-se um teste de cointegração usando o método de Johansen (1988). Em um terceiro passo, estima o modelo vetorial de correção de erro com threshold, determinando o valor de $\gamma$ e teste de não linearidade usando o procedimento Hansen e Seo (2002).

A estimação do modelo conforme Hansen e Seo (2002) consistem em: i) formar uma grade entre os candidatos a vetor de cointegração $\beta$ e os valores possíveis de threshold $\gamma$; ii) para cada valor de $(\beta, \gamma)$ na grade, calcular o restante dos parâmetros do 
p. 63 - Integração de preços nas principais praças de comercialização do milho em grão no Brasil

modelo por máxima verossimilhança; iii) Encontrar $(\hat{\beta}, \hat{\gamma})$ como os valores de $(\beta, \gamma)$ sobre a grade na qual produz o menor valor de $\log |\widehat{\Sigma}(\beta, \gamma)|$; iv) gerar o valor dos parâmetros do modelo a partir do valor encontrado do $\operatorname{par}(\hat{\beta}, \hat{\gamma})$. O teste de não-linearidade testa a hipótese nula de cointegração linear contra a hipótese alternativa de cointegração com threshold, através do computo dos $p$-valores.

\section{RESULTADOS E DISCUSSÃO}

Uma série temporal de dados sobre custos de transação dificilmente são disponibilizados e, portanto, um estudo de integração de mercado que não considere tais custos pode não ter resultados esperados ou não confiáveis. Assim, neste artigo é aplicado um modelo vetorial de correção de erro com threshold para cada par de mercados.

Antes da estimação do TVEC é feito o teste de raiz unitária das séries de preços por meio do método ADF. O resultado do teste ADF identifica a presença de raiz unitária em todas as séries de preço do milho. Em todos os casos das séries em nível, a hipótese nula de presença de raiz unitária não foi rejeitada aos níveis de significância de 1\%, 5\% e $10 \%$. Porém, para as séries diferenciadas, rejeitou-se a hipótese nula de raiz unitária, pois os valores da estatística $\tau$ são menores que os valores críticos aos mesmos níveis de significância. Portanto, as séries de preços do milho em primeira diferença são estacionárias.

O teste de cointegração através da metodologia de Johansen por meio da estatística do teste traço rejeita a hipótese nula de nenhum vetor de cointegração. Nesse caso, o valor do teste traço é maior que os valores críticos encontrados. Dessa forma, cada um dos mercados físicos com relação ao mercado de Campinas possui um equilíbrio de longo prazo entre eles.

Tabela 1 - Teste de cointegração de Johansen: análise entre Campinas e demais mercados - teste traço

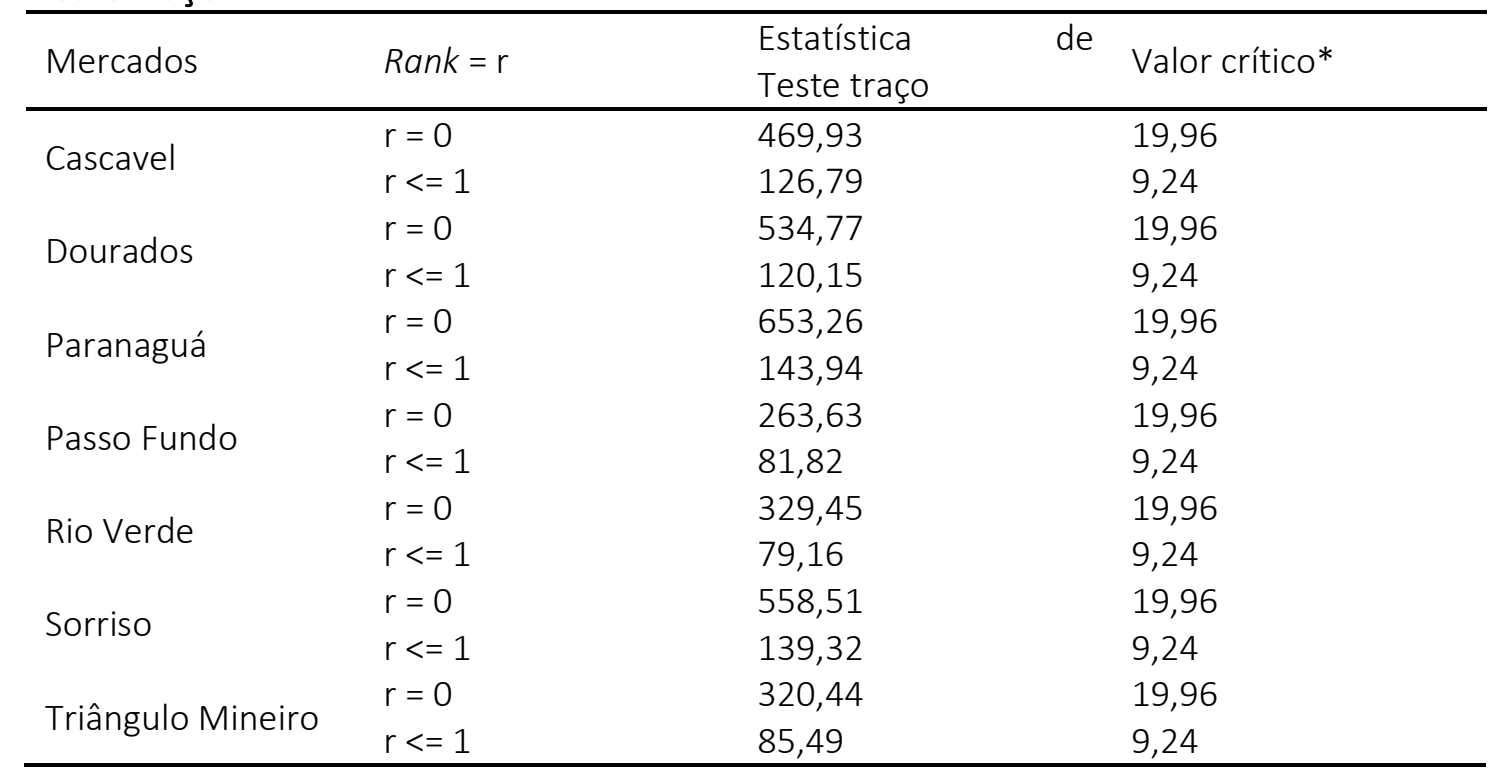

*Rejeição da hipótese nula a 5\% de significância. Fonte: Resultados da pesquisa 
O passo seguinte é estimar o modelo TVEC. Os resultados da Tabela 2 mostram os valores de threshold obtidos entre cada região com relação a Campinas. O valor de cada threshold dá indícios da magnitude dos custos de transação envolvidos entre as regiões. Nesse sentido, um choque de preços resultado por excesso de demanda em Campinas, por exemplo, superior a 1,22\%; 1,45\%, 1,26\%, 0,9\%; 1,27\%; 1,03\% e 1,39\% promovem um ajustamento de preços em Cascavel, Dourados, Paranaguá, Passo Fundo, Rio Verde, Sorriso e Triângulo Mineiro, respectivamente.

A partir do valor threshold são determinados dois regimes, Down e Up. A separação dos regimes é obtida pela multiplicação do logaritmo natural do preço médio do milho com o valor do threshold. Relacionado aos regimes, o parâmetro threshold pode apresentar sinais positivo ou negativo. Quando o valor do parâmetro é positivo significa que a maior parte das observações totais se encontra no regime Down. Do contrário quando o valor do parâmetro é negativo significa que a maior parte das observações totais do modelo se encontra no regime Up.

Tabela 2 - Resultados do modelo TVEC bivariado para todas as praças, considerando Campinas como mercado de referência, de 2 de Janeiro de 2009 a 29 de Dezembro de 2016.

\begin{tabular}{|c|c|c|c|c|c|c|}
\hline \multirow{2}{*}{ Praças } & \multirow{2}{*}{ Threshold } & \multicolumn{2}{|c|}{ Regime Down } & \multicolumn{2}{|c|}{ Regime Up } & \multirow{2}{*}{$\begin{array}{l}\text { Teste de } \\
\text { Hansen } \\
\text { e Seo }\end{array}$} \\
\hline & & ECT praça & ECT campinas & ECT praça & ECT campinas & \\
\hline Cascavel & $-0,0122941$ & $\begin{array}{l}-0,0022 \\
(0,9926)^{\mathrm{NS}}\end{array}$ & $\begin{array}{l}-1,2437 \\
(0,000)^{* * *}\end{array}$ & $\begin{array}{l}0,3109 \\
(0,000)^{* * *}\end{array}$ & $\begin{array}{l}-0,6648 \\
(0,000)^{* * *}\end{array}$ & 0,04 \\
\hline Dourados & 0,014555 & $\begin{array}{l}0,4688 \\
(0,000)^{* * *}\end{array}$ & $\begin{array}{l}-0,5502 \\
(0,000)^{* * *}\end{array}$ & $\begin{array}{l}0,6824 \\
(0,0794)^{*}\end{array}$ & $\begin{array}{l}-0,5858 \\
(0.0011)^{* *}\end{array}$ & 0,50 \\
\hline Paranaguá & $-0,0126578$ & $\begin{array}{l}0,6937 \\
(0,0059)^{* *}\end{array}$ & $\begin{array}{l}-0,1404 \\
(0,2986)^{N S}\end{array}$ & $\begin{array}{l}-0,3365 \\
(0,000)^{* * *}\end{array}$ & $\begin{array}{l}-0,2927 \\
(0,000)^{* * *}\end{array}$ & 0,82 \\
\hline Passo Fundo & $-0,009013$ & $\begin{array}{l}0,7964 \\
(0,000)^{* * *}\end{array}$ & $\begin{array}{l}-0,4711 \\
(0,0011)^{* *}\end{array}$ & $\begin{array}{l}0,3042 \\
(0,000)^{* * *}\end{array}$ & $\begin{array}{l}-0,4713 \\
(0,000)^{* * *}\end{array}$ & 0,53 \\
\hline Rio Verde & $-0,0127183$ & $\begin{array}{l}1,1038 \\
(0,0030)^{* *}\end{array}$ & $\begin{array}{l}-0,0806 \\
(0,7117)^{\mathrm{NS}}\end{array}$ & $\begin{array}{l}0,4916 \\
(0,000)^{* * *}\end{array}$ & $\begin{array}{l}-0,5648 \\
(0,000)^{* * *}\end{array}$ & 0,01 \\
\hline Sorriso & $-0,0103794$ & $\begin{array}{l}0,1190 \\
(0,7423)^{\mathrm{NS}}\end{array}$ & $\begin{array}{l}-0,2445 \\
(0,0475)^{*}\end{array}$ & $\begin{array}{l}0,5561 \\
(0,000)^{* * *}\end{array}$ & $\begin{array}{l}-0,4745 \\
(0,000)^{* * *}\end{array}$ & 0,01 \\
\hline Triângulo Mineiro & 0,0139129 & $\begin{array}{l}0,4396 \\
(0,000)^{* * *}\end{array}$ & $\begin{array}{l}-0,6668 \\
(0,000)^{* * *}\end{array}$ & $\begin{array}{l}0,0192 \\
(0,9548)^{\mathrm{NS}}\end{array}$ & $\begin{array}{l}-0,4993 \\
(0,0382)^{*}\end{array}$ & 0,08 \\
\hline
\end{tabular}

Nota: * Significativo a $5 \%$; ${ }^{* *}$ significativo a $1 \% ; * * *$ significativo a $0,01 \%$; NS - Não significativo. Fonte: Resultados da pesquisa.

No primeiro modelo TVEC envolvendo Cascavel e Campinas o valor encontrado para o parâmetro threshold, em módulo, foi de 0,0122941 no período analisado. A partir do parâmetro threshold é definido dois regimes. No regime Down é definido pelas observações cujo desvio do equilíbrio de longo prazo é inferior a 0,0122941. Em valores financeiros (R\$) utilizando o preço médio do milho em Cascavel (R\$ 24,96), tem-se que desvios inferiores a $\mathrm{R} \$ 0,04$ (preços inferiores a $\mathrm{R} \$ 24,92$ ) não causam nenhum processo de ajustamento de preços. Com relação a erro equilibrador dos desvios de longo prazo, 
p. 65 - Integração de preços nas principais praças de comercialização do milho em grão no Brasil

este não é estatisticamente significativo mostrando que nesse regime os preços se ajustam simultaneamente. Essa região contém 7,4\% das observações. No regime Up é encontrado as observações na qual os desvios, em módulo, são superiores ao valor do threshold de 0,0122941. Assim, em valores financeiros os desvios superiores a $\mathrm{R} \$ 0,04$ (preços superiores a $\mathrm{R} \$ 25,00$ ) induziram processos de ajustamento de preços. O erro equilibrador nesse regime é estatisticamente significativo a $0,01 \%$ e cujo valor encontrado é 0,3109; indicando que a diferença de 31,09\% entre os preços de Cascavel e Campinas são corrigidos diariamente em pouco mais de 3 dias. Essa região contém $92,6 \%$ das observações.

No segundo modelo envolvendo Dourados e Campinas o valor encontrado para o parâmetro threshold foi de 0,014555 no período analisado. Analisando os preços da região de Dourados, no regime Down as observações cujo desvio do equilíbrio de longo prazo, em módulo, é inferior a 0,014555. Em valores financeiros (R\$) utilizando o preço médio do milho em Dourados ( $R \$ 21,74)$, tem-se que desvios inferiores a $R \$ 0,04$ (preços inferiores a $\mathrm{R} \$ 21,70)$ não causam nenhum processo de ajustamento de preços. Com relação a erro equilibrador dos desvios de longo prazo, este é estatisticamente significativo com valor de 0,4688 , mostrando que a diferença de $46,88 \%$ entre os preços de Dourados e Campinas são corrigidos em 2 dias. Essa região contém 94,4\% das observações. No regime Up é encontrado as observações na qual os desvios, em módulo, são superiores ao valor do threshold de 0,014555. Assim, em valores financeiros os desvios superiores a $\mathrm{R} \$ 0,04$ (preços superiores a $\mathrm{R} \$ 21,78$ ) induziram processos de ajustamento de preços. O erro equilibrador nesse regime é estatisticamente significativo a $5 \%$ e cujo valor encontrado é 0,6824 , indicando que a diferença de $68,24 \%$ entre os preços de Dourados e Campinas são corrigidos diariamente em pouco mais de 1 dia. Essa região contém $5,6 \%$ das observações.

No terceiro modelo envolvendo Paranaguá e Campinas o valor encontrado para o parâmetro threshold foi de $-0,0126578$ no período analisado. No regime Down é encontrado as observações cujo desvio do equilíbrio de longo prazo, em módulo, é inferior a 0,0126578. Em valores financeiros (R\$) utilizando o preço médio do milho em Paranaguá ( $R \$ 27,92)$, tem-se que desvios inferiores a $R \$ 0,04$ (preços inferiores a $R \$$ $27,88)$ não causam nenhum processo de ajustamento de preços. Com relação ao erro equilibrador dos desvios de longo prazo, este é estatisticamente significativo a $1 \% \mathrm{com}$ valor de 0,6937 mostrando que a diferença de 69,37\% entre os preços de Paranaguá e Campinas são corrigidos em menos de 2 dias. Essa região contém 5,8\% das observações totais. No regime Up é encontrado as observações na qual os desvios, em módulo, são superiores ao valor do threshold de 0,0126578. Assim, em valores financeiros os desvios superiores a $\mathrm{R} \$ 0,04$ (preços superiores a $\mathrm{R} \$ 27,96$ ) induziram processos de ajustamento de preços. O erro equilibrador nesse regime é estatisticamente significativo a 0,01\% e cujo valor encontrado é 0,3365, indicando que a diferença de 33,65\% entre os preços de Paranaguá e Campinas são corrigidos diariamente em aproximadamente 3 dias. Essa região contém 94,2\% das observações.

No quarto modelo envolvendo Passo Fundo e Campinas o valor encontrado para o parâmetro threshold, em módulo, foi de 0,009013 no período analisado. Sendo assim, no regime Down os desvios do equilíbrio de longo prazo inferior a 0,009013 não 
induziram processo de ajustamento, ou seja, em valores financeiros, utilizando o preço médio do milho em Passo Fundo ( $R \$ 27,30$ ), os desvios inferiores a $R \$ 0,03$ (preços inferiores a $\mathrm{R} \$ 27,27)$ não causam nenhum processo de ajustamento de preços. Acerca do erro equilibrador dos desvios de longo prazo, este é estatisticamente significativo a 0,01\% com valor de 0,7964 , de modo que a diferença de 79,64\% entre os preços de Passo Fundo e Campinas são corrigidos em pouco mais de 1 dia. Essa região contém 14,1\% das observações totais. Já no regime Up os desvios no equilíbrio de longo prazo levaram a um processo de ajustamento, o que significa que os desvios superiores a $\mathrm{R} \$ 0,03$ (preços superiores a $\mathrm{R} \$ 27,33$ ) induziram processos de ajuste nos preços. $\mathrm{O}$ erro equilibrador nesse regime é estatisticamente significativo a 0,01\% e cujo valor encontrado é 0,3042, indicando que a diferença de $30,42 \%$ entre os preços de Passo Fundo e Campinas são corrigidos diariamente em pouco mais de 3 dias. Essa região contém 85,9\% das observações.

No quinto modelo TVEC bivariado envolvendo as regiões de Rio Verde e Campinas o valor encontrado para o parâmetro threshold, em módulo, foi de 0,0127183. Assim, levando em conta o preço médio em Rio Verde $(R \$ 23,09)$, tem-se que desvios inferiores a $R \$ 0,04$ não causam nenhum processo de ajustamento, isto é, valores menores que $R \$$ 23,05 se encontram no regime Down. Acerca do erro equilibrador, observa-se que é estatisticamente significativo a $1 \%$ com valor de 1,1038; o que significa que a diferença de 110,38\% entre os preços de Rio Verde e Campinas são corrigidos em menos de 1 dia. Essa região contém 7,6\% das observações totais. Já no regime Up os desvios no equilíbrio de longo prazo levaram a um processo de ajustamento, ou seja, os desvios superiores a $\mathrm{R} \$ 0,04$ (preços superiores a $\mathrm{R} \$ 23,13$ ) induziram processos de ajuste nos preços. O erro equilibrador nesse regime é estatisticamente significativo a $0,01 \%$ e cujo valor encontrado é 0,4916 , indicando que a diferença de 49,16\% entre os preços de Rio Verde e Campinas são corrigidos diariamente em aproximadamente 2 dias. Essa região contém $92,4 \%$ das observações.

No sexto modelo TVEC bivariado envolvendo as regiões de Sorriso e Campinas o valor encontrado para o parâmetro threshold, em módulo, foi de 0,0103794. Assim, levando em conta o preço médio do milho em Sorriso (R\$ 15,98), tem-se que desvios inferiores a $\mathrm{R} \$ 0,03$ não causam nenhum processo de ajustamento, isto é, valores menores que $\mathrm{R} \$ 15,95$ que se encontram no regime Down. Acerca do erro equilibrador, nota-se que é não significativo estatisticamente, o que significa que os preços de Sorriso e Campinas se ajustam simultaneamente. Essa região contém 11,9\% das observações totais. Já no regime Up os desvios no equilíbrio de longo prazo levaram a um processo de ajustamento, ou seja, os desvios superiores a $R \$ 0,03$ (preços superiores a $R \$ 16,01$ ) induziram processos de ajuste nos preços. O erro equilibrador nesse regime é estatisticamente significativo a 0,01\% e cujo valor encontrado é 0,5561, indicando que a diferença de 55,61\% entre os preços de Sorriso e Campinas são corrigidos diariamente em aproximadamente 2 dias. Essa região contém 88,1\% das observações.

No último modelo TVEC bivariado envolvendo as regiões do Triângulo Mineiro e Campinas o valor encontrado para o parâmetro threshold, em módulo, foi de 0,0139129. Assim, levando em conta o preço médio do milho no Triângulo Mineiro (R\$25,41), temse que desvios inferiores a $R \$ 0,05$ não causam nenhum processo de ajustamento, isto é, 
p. 67 - Integração de preços nas principais praças de comercialização do milho em grão no Brasil

valores menores que $\mathrm{R} \$ 25,36$ se encontram no regime Down. Acerca do erro equilibrador, observa-se que é estatisticamente significativo a 0,01\% com valor de 0,4396; o que significa que a diferença de 43,96\% entre os preços do Triângulo Mineiro e Campinas são corrigidos diariamente em pouco mais de 2 dias. Essa região contém $93,6 \%$ das observações totais. Já no regime Up os desvios no equilíbrio de longo prazo levaram a um processo de ajustamento, ou seja, os desvios superiores a $\mathrm{R} \$ \mathbf{0 , 0 5}$ (preços superiores a $\mathrm{R} \$ 25,46$ ) induziram processos de ajuste nos preços. O erro equilibrador nesse regime não é estatisticamente significativo, o que significa que os preços no Triângulo Mineiro e Campinas se ajustam simultaneamente. Essa região contém 6,4\% das observações.

Os resultados do modelo TVEC com dois regimes mostram que nos mercados de Cascavel, Paranaguá, Passo Fundo, Rio Verde e Sorriso a maioria de suas observações se encontram no regime Up, onde ocorrem choques maiores que o valor de threshold. Enquanto nos mercados de Dourados e Triângulo Mineiro a maior parte das observações se localizam no regime Down, onde os valores inferiores ao threshold não induziram a um processo de ajustamento, pois os preços responderam apenas as variações dos preços passados.

Na última coluna da Tabela 2 observam-se os resultados do teste de Hansen e Seo (2002). A hipótese nula é de linearidade e a hipótese alternativa é de não linearidade. Com base nisso é rejeitado a hipótese nula para os mercados de Cascavel, Rio Verde, Sorriso e Triângulo Mineiro, mostram que estes se ajustam melhor ao modelo TVEC. Porém, para os mercados de Dourados, Paranaguá e Passo Fundo não se rejeita a hipótese nula de linearidade.

\section{CONSIDERAÇÕES FINAIS}

Nos últimos anos o mercado do milho em grão tem apresentado mudanças na estrutura de oferta e demanda. A produção vem crescendo, estimulada pelo aumento da demanda interna e externa. De maneira que o milho é o segundo grão mais cultivado, somente atrás da cultura da soja. Onde um dos principais destinos é a indústria de ração animal, o outro para consumo humano, que é bastante utilizado como insumo na produção de cerveja, refrigerantes, farinha, biscoitos, doces, etc.

O objetivo geral desse artigo foi analisar a integração de preços no mercado de milho no Brasil. Foram considerados os mercados físicos de Campinas, Cascavel, Paranaguá, Passo Fundo, Rio Verde, Sorriso, Dourados e Triângulo Mineiro, regiões de maior dinâmica na comercialização do milho.

Os testes econométricos foram realizados utilizando preços diários do milho no período de janeiro de 2009 a dezembro de 2016. Buscou-se verificar a ordem de integração das séries, a quantidade de vetores de cointegração existente e os processos de ajuste de preços no curto e longo prazo.

A identificação de presença de raiz unitária foi realizada utilizando o teste ADF e os resultados mostram que as séries de preços são não estacionárias em nível. No 
entanto, em primeira diferença, as séries se tornaram estacionárias, ou seja, sem a presença de raiz unitária. Dessa forma, integradas de ordem um.

Entre todas as regiões analisadas considerou-se Campinas como mercado de referência para formação de pares de preços e prosseguir com os testes econométricos. O teste de cointegração de Johansen mostrou a presença de ao menos um vetor de cointegração para cada par de preços, indicando uma relação de equilíbrio de longo prazo. O modelo TVEC estimado para cada par de preços identificou um parâmetro threshold, indicando presença de custo de transação entre os mercados. Assim, os desequilíbrios de longo prazo existentes são corrigidos num processo de ajustamento de preço que leva em torno de 2 a 3 dias.

Com relação aos regimes de ajustamento, a maior parte das observações para mercados de Dourados e Triângulo Mineiro se encontram no regime Down, onde os desvios do equilíbrio de longo prazo são menores que o valor do threshold. Enquanto, a maior parte das observações para os mercados de Cascavel, Paranaguá, Passo Fundo, Rio Verde e Sorriso se encontra no regime Up. Onde os desequilíbrios de longo prazo são maiores que o valor do threshold, situação em que ocorre um processo de ajustamento de preços.

O mercado do milho se mostra bastante integrado com a região de Campinas, pois o custo de transação existente é baixo, sugerindo deslocamento do grão. Dentre as regiões analisadas, Dourados, Triângulo Mineiro e Rio Verde apresentaram um valor threshold (custo de transação) maior. Uma provável explicação é que as regiões do Triângulo Mineiro e Rio Verde podem ter uma demanda local significativos, reduzindo o fluxo inter-regiões do grão. Aliás, houve também uma mudança na legislação referente à cobrança do ICMS nas operações de exportação de milho em Goiás, beneficiando o consumo local ao aumentar a tributação sobre o milho exportado. No caso de Dourados é possível que sua produção se destine a outras regiões com alta demanda do grão, como Paraná e Rio Grande do Sul. Em geral, o mercado é bem dinâmico com relação a Campinas, na qual influência de maneira intensiva e rápida as variações de preços em outras regiões.

O estudo de integração de mercado do milho fornece informações importantes sobre o comportamento dos preços e a magnitude dos custos de transação existentes e até que ponto pode interferir na transmissão dos preços entre as regiões. E como sugestão de pesquisas futuras é indicada utilizar um modelo TVEC com três regimes e incluir os preços do milho no mercado internacional, além de estender a análise para outras regiões.

\section{REFERÊNCIAS}

ABIMILHO - Associação Brasileira das Indústrias do Milho. Estatísticas. Disponível em: <http://www.abimilho.com.br/estatisticas>. Acesso em: 12 set. 2016.

Notícias.

Disponíveis

em:

<http://www.abramilho.org.br/noticias.php?cod=3825>. Acesso em: 18 jul. 2016. 
p. 69 - Integração de preços nas principais praças de comercialização do milho em grão no Brasil

ALVES, J. da S.; LIMA, R. C. Integração e mercados espacialmente separados de etanol no Brasil: uma análise considerando efeitos threshold. In: Encontro de economia baiana. 6, 2010, Salvador. Anais... Salvador, 2010. 346-368.

ALVES, J. da S. Transmissão de preços de açúcar e álcool em mercados espacialmente separados no Brasil na presença de custos de transação. 138 f. Tese (Doutorado) Universidade Federal de Pernambuco. CCSA. Economia, Recife, 2009.

BALKE, N. S.; FOMBY, T. B. Threshold cointegration. International Economic Review, v. 38, n. 3, p. 627-645, 1997.

BALCOMBE, K.; BAILEY, A.; BROOKS, J. Threshold effects in price transmission: The case of Brazilian wheat, maize, and soya prices. American Journal of Agricultural Economics, v. 89, n. 2, p. 308-323, maio 2007.

BRASIL. Ministério de Agricultura, Pecuária e Abastecimento. Projeções do agronegócio: Brasil 2015/16 a 2025/2026. Assessoria de Gestão Estratégica, Brasília: Mapa/ACS, 2016.

CAMPENHOUT, B. V. Modelling trends in food market integration: method and an application to Tanzanian maize markets. Food Policy, v. 32, p. 112-127, 2007.

COASE, R. H. The nature of the firm. Economica, v. 4, n. 16, 1937.

CONAB - Companhia Nacional de Abastecimento. Perspectiva para a agropecuária. Brasília, v. 1, 2015.

Acompanhamento da safra brasileira de grãos: quinto levantamento. Brasília, v. 4, n. 5, 2017.

CUNHA, C. A. da; SILVA NETO, W. A. da; SCALCO, P. R.; Transmissão assimétrica nos preços do milho: o caso do Centro-Oeste. In: 52ㅇ Congresso Brasileiro de Economia, Administração e Sociologia Rural. Goiânia, 2014. Anais..., Goiânia, 2014.

CUNHA, C. A. da; SCALCO, P. R.; WANDER, A. E. Custos de transação e comportamento da base para o preço do milho em Rio Verde, GO. Revista de Política Agrícola, v. 22, n. 3, p. 88-95, jul./set. 2013.

DICKEY, D. A.; FULLER, W. A. Distribution of the Estimators for Autoregressive Time Series with a Unit Root. Journal of the American Statistical Association, v. 74, p. 427-431, 1979.

FACKLER, P. L.; GOODWIN, B. K. Spatial price analysis. In: RAUSSER, G. C.; GARDEN, B. L. (Org). Handbook of Agricultural Economics, p. 971-1025, 2001.

FIANI, R. Teoria dos custos de transação. In: KUPFER, D.; HASENCLEVER, L. Economia Industrial: fundamentos teóricos e práticas no Brasil. Rio de Janeiro: Campus, 2002.

GONZALEZ-RIVERA, G.; HELFAND, S. M. The extent, pattern and degree of market integration: A multivariate approach for the Brazilian rice market. American Journal of Agricultural Economics, v. 83, p. 576-592, 2001.

GOODWIN, B. K.; PIGGOTT, N. E. Spatial market integration in the presence of threshold effects. American Journal of Agricultural Economics, v. 83, p. 302 317, 2001.

HANSEN, B. E; SEO, B. Testing for two-regimes threshold cointegration in vector errorcorrection models. Journal of Econometrics, v.110, n. 9, p. 293-318, 2002. 
JOHANSEN, S. Statistical analysis of cointegration vectors. Journal of Economic Dynamics and Control, v. 12, p. 231-254, 1988.

JOHANSEN, S.; JUSELIUS, K. Maximum likelihood estimation and inference on cointegration - with application to the demand for money. Oxford Bulletin on Economics and Statistics, v. 52, n. 1, p. 169-210, 1990.

JUSTEN JUNIOR, A. A.; VIEIRA, K. M.; CORONEL, D. A. Eficiência dos mercados da soja no Brasil (2004-2010). Organizações Rurais \& Agroindustriais, v. 15, n. 2, p. 180-193, 2013.

MACHADO, G. R.; WANDER, A. E.; ARÊDES, A.; SILVA, F. H. F. O Ambiente Institucional Formal e seu Impacto na Competitividade do SAG da Carne Bovina: uma Análise do Programa de Rastreabilidade da Carne Bovina Brasileira. In: 48 CONGRESSO BRASILEIRO DE ECONOMIA E SOCIOLOGIA RURAL. Campo Grande, 2010. Anais..., Campo Grande, 2010. p. 132-147.

MATTOS, L. B. Efeitos de custos de transação sobre a integração espacial de mercados regionais de carne de frango no Brasil. 162 f. 2008. Tese (Doutorado) - Universidade Federal de Viçosa. Viçosa, MG, 2008.

MATTOS, L. B.; LIMA, J. E.; LIRIO, V. S. Integração espacial de mercados na presença de custos de transação: um estudo para o mercado de boi gordo em Minas Gerais e São Paulo. Revista de Economia e Sociologia Rural, v. 47, n. 1, jan./mar. 2009.

MATTOS, L. B.; LÍRIO, V. S.; LIMA, J. E.; CAMPOS, A. C. Modelos de cointegração com um ou dois limiares: uma aplicação para o preço do frango inteiro resfriado em mercados atacadistas no Brasil. Revista de Economia e Sociologia Rural, v. 48, n. 4, p. 597-617, out./dez. 2010.

MEYER, J. Measuring Market integration in the presence of transaction costs: A threshold vector error correction approach. Agricultural Economics, v. 31, n. 21, p. 327-334, 2004.

PEREIRA, L. R. R. Integração espacial no mercado brasileiro de boi gordo. 166 p. Tese (Doutorado em Economia Aplicada) - Universidade Federal de Viçosa, 2005.

ROSADO, P. L. Integração espacial entre os mercados brasileiro de suínos. 117 f. 2006. Tese (Economia Aplicada) - Pós-Graduação em Economia Aplicada, Universidade Federal de Viçosa, Viçosa, 2006.

SEPHTON, P. S. Spatial Market arbitrage and threshold cointegration. American Journal of Agricultural Economics, v. 85, n 4, p. 1041-1046, 2003.

SOUSA, E. P.; BRAGA, M. J.; CUNHA, D. A. Interdependência dos preços do milho no sul brasileiro. Revista de Economia, v. 36, n. 2 (ano 34), p. 71-90, maio/ago. 2010.

TARDELLI, B. L. S. Integração espacial da soja escoada do Mato Grosso para o porto de Santos: Uma análise sob custos de transação. In: Encontro Nacional de Economia. 40, 2012, Porto de Galinhas. Anais..., 2012. p. 1-17.

WILLIAMSON, O. E. The mechanisms of governance. New York, New York: Oxford University Press, 1996.

ZYLBERSZTAJN, D.; NEVES, M. F. Economia e negócio agroindustrial. São Paulo: Pioneira, 2000. 